COLBY-96-04

IUHET 338

July 1996

\title{
WAVE-PACKET REVIVALS FOR QUANTUM SYSTEMS WITH NONDEGENERATE ENERGIES
}

\author{
Robert Bluhm, ${ }^{a}$ V. Alan Kostelecký, ${ }^{b}$ and Bogdan Tudose ${ }^{b}$ \\ ${ }^{a}$ Physics Department \\ Colby College \\ Waterville, ME 04901, U.S.A. \\ ${ }^{b}$ Physics Department \\ Indiana University \\ Bloomington, IN 47405, U.S.A.
}

\begin{abstract}
The revival structure of wave packets is examined for quantum systems having energies that depend on two nondegenerate quantum numbers. For such systems, the evolution of the wave packet is controlled by two classical periods and three revival times. These wave packets exhibit quantum beats in the initial motion as well as new types of long-term revivals. The issue of whether fractional revivals can form is addressed. We present an analytical proof showing that at certain times equal to rational fractions of the revival times the wave packet can reform as a sum of subsidiary waves and that both conventional and new types of fractional revivals can occur.
\end{abstract}

Accepted for publication in Physics Letters A 
Among the distinctive quantum features of wave-packet evolution in a potential is the formation of fractional revivals. These appear when the packet reforms as a sum of distinct subsidiary waves some time after its initial collapse. Fractional revivals have been observed experimentally in the motion of atomic Rydberg wave packets [1, 2, 3] and in molecular vibrational wave packets [4]. In these systems, the wave packet is formed via excitation with a short laser pulse, and its motion is detected using a pump-probe configuration [5]. The revival structure of a free hydrogenic wave packet is determined by the dependence of the component-eigenstate energies on the principal quantum number. Similarly, the vibrational states of a molecular wave packet depend only on the vibrational quantum number.

For the case of quantum systems with energies depending only on one quantum number $n$, a generic proof has been given showing that fractional revivals form [6]. The proof applies to packets that are a superposition of states strongly peaked around a central value $\bar{n}$ of $n$. Initially, the motion of a wave packet of this type is periodic with period $T_{\mathrm{cl}}$ equal to the period of the corresponding classical motion. After several classical periods, the wave packet spreads and collapses. The subsequent formation of revivals is characterized by a time scale $t_{\text {rev }}$ called the revival time. Fractional revivals occur at times that are rational fractions of $t_{\text {rev }}$. The associated subsidiary wave packets exhibit a periodicity in their motion equal to a fraction of $T_{\mathrm{cl}}$.

A full revival can be viewed as a special fractional revival occurring when the wave packet reforms as a single entity evolving with the classical periodicity. For quantum systems with energies that are quadratic in $n$, the full revivals are perfect in the sense that the wave function at time $t=t_{\text {rev }}$ is exactly equal to the wave function at $t=0$ [7]. For other systems the revivals at $t=t_{\text {rev }}$ are imperfect, and new structure emerges after several cycles of collapse and revival. A detailed analysis involves introducing a superrevival time scale $t_{\mathrm{sr}}$ and showing that the interplay between $t_{\mathrm{sr}}$ and $t_{\mathrm{rev}}$ results in a new sequence of fractional and full superrevivals for $t \gg t_{\mathrm{rev}}$ [8]. Among these, on time scales much larger than the superrevival time scale $t_{\mathrm{sr}}$, are the long-term revivals already predicted in early work on Rydberg wave packets [9] that appear when the phase in the wave function is an integer multiple of $2 \pi$ [10.

The above analyses can be applied provided the physics of the wave packet is 
controlled by a single quantum number. As an example, consider a radial Rydberg wave packet in an alkali-metal atom. The energies of the atom have quantum defects and hence depend on more than one quantum number. However, for large enough principal quantum number and fixed angular-momentum quantum number, the quantum defects are constant to an excellent approximation. The fractional revival and superrevival structure of a wave packet formed as a superposition of, for example, $\mathrm{p}$ states can therefore be treated using the above methods [8] taking the quantum-defect shifts into account analytically [11]. A theoretical description of these wave packets as squeezed states can be used to extend the treatment to elliptical wave packets in alkali-metal atoms [12].

To study more complicated cases, such as Rydberg wave packets in external fields, an analysis is required that allows for nondegenerate energies depending on more than one quantum number. This is the topic of the present paper.

We focus attention here on the dynamics of a wave packet $\Psi(t)$ formed as a coherent superposition of states $\phi_{n_{1} n_{2}}$ with energies $E_{n_{1} n_{2}}$ depending on two quantum numbers $n_{1}$ and $n_{2}$ :

$$
\Psi(t)=\sum_{n_{1}, n_{2}} c_{n_{1} n_{2}} \phi_{n_{1} n_{2}} \exp \left[-i E_{n_{1} n_{2}} t\right]
$$

Here, $c_{n_{1} n_{2}}$ are complex weighting coefficients. Note that $n_{1}$ and $n_{2}$ may represent effective quantum numbers in, for instance, a perturbative treatment, for which the states $\phi_{n_{1} n_{2}}$ may be only approximate energy eigenstates. Our analysis in the present work is therefore relevant for experiments studying the behavior of Rydberg wave packets in external fields, where the energy degeneracy in the superposition of states is lifted [13, 14, 15, 16, 17].

We suppose that the superposition is weighted around central values $\bar{n}_{1}$ and $\bar{n}_{2}$ of the two quantum numbers. The weighting coefficients $c_{n_{1} n_{2}}$ are therefore taken to be strongly peaked around a central value of the energy $E_{\bar{n}_{1} \bar{n}_{2}}$, as would result from an excitation process using short laser pulses. The energy can then be expanded in a Taylor series as

$$
E_{n_{1} n_{2}} \simeq E_{\bar{n}_{1} \bar{n}_{2}}+\left(\frac{\partial E}{\partial n_{1}}\right)_{\bar{n}_{1}, \bar{n}_{2}}\left(n_{1}-\bar{n}_{1}\right)+\left(\frac{\partial E}{\partial n_{2}}\right)_{\bar{n}_{1}, \bar{n}_{2}}\left(n_{2}-\bar{n}_{2}\right)
$$




$$
\begin{gathered}
+\frac{1}{2}\left(\frac{\partial^{2} E}{\partial n_{1}^{2}}\right)_{\bar{n}_{1}, \bar{n}_{2}}\left(n_{1}-\bar{n}_{1}\right)^{2}+\frac{1}{2}\left(\frac{\partial^{2} E}{\partial n_{2}^{2}}\right)_{\bar{n}_{1}, \bar{n}_{2}}\left(n_{2}-\bar{n}_{2}\right)^{2} \\
+\left(\frac{\partial^{2} E}{\partial n_{1} \partial n_{2}}\right)_{\bar{n}_{1}, \bar{n}_{2}}\left(n_{1}-\bar{n}_{1}\right)\left(n_{2}-\bar{n}_{2}\right)+\ldots
\end{gathered}
$$

Since the energy depends on two variables, the expansion contains a mixed derivative term at second order.

When Eq. (2) is substituted into (1) and an irrelevant overall phase is disregarded, it follows that each derivative term corresponds to a distinct time scale. We define

$$
\begin{gathered}
T_{\mathrm{cl}}^{(1)}=\frac{2 \pi}{\left(\frac{\partial E}{\partial n_{1}}\right)_{\bar{n}_{1}, \bar{n}_{2}}} \quad, \quad T_{\mathrm{cl}}^{(2)}=\frac{2 \pi}{\left(\frac{\partial E}{\partial n_{2}}\right)_{\bar{n}_{1}, \bar{n}_{2}}}, \\
t_{\mathrm{rev}}^{(1)}=\frac{2 \pi}{\frac{1}{2}\left(\frac{\partial^{2} E}{\partial n_{1}^{2}}\right)_{\bar{n}_{1}, \bar{n}_{2}}}, \quad t_{\mathrm{rev}}^{(2)}=\frac{2 \pi}{\frac{1}{2}\left(\frac{\partial^{2} E}{\partial n_{2}^{2}}\right)_{\bar{n}_{1}, \bar{n}_{2}}}, \\
t_{\mathrm{rev}}^{(12)}=\frac{2 \pi}{\left(\frac{\partial^{2} E}{\partial n_{1} \partial n_{2}}\right)_{\bar{n}_{1}, \bar{n}_{2}}}
\end{gathered}
$$

where we have assumed for simplicity that the quantum numbers take integer-spaced values. For each quantum number, there is a classical period and a revival time scale. In addition, the mixed derivative term generates a third time scale $t_{\mathrm{rev}}^{(12)}$, which we call the cross-revival time. This term has no analog in the conventional revival structure of free Rydberg wave packets. Keeping terms to second order then gives for $\Psi(t)$ the expression

$$
\Psi(t)=\sum_{k_{1}, k_{2}} c_{k_{1} k_{2}} \phi_{k_{1} k_{2}} \exp \left[-2 \pi i\left(\frac{k_{1} t}{T_{\mathrm{cl}}^{(1)}}+\frac{k_{2} t}{T_{\mathrm{cl}}^{(2)}}+\frac{k_{1}^{2} t}{t_{\mathrm{rev}}^{(1)}}+\frac{k_{2}^{2} t}{t_{\mathrm{rev}}^{(2)}}+\frac{k_{1} k_{2} t}{t_{\mathrm{rev}}^{(12)}}\right)\right]
$$

where $k_{1}=\left(n_{1}-\bar{n}_{1}\right), k_{2}=\left(n_{2}-\bar{n}_{2}\right)$, and where the coefficients $c_{k_{1} k_{2}}$ and eigenstates $\phi_{k_{1} k_{2}}$ are related to the analogous quantities in Eq. (1) by shifts in the subscripts.

Consider the time evolution of the wave packet. When $t$ is small, the dominant terms in the time-dependent phase in Eq. (6) are the first two. They produce beating between the classical periods $T_{\mathrm{cl}}^{(1)}$ and $T_{\mathrm{cl}}^{(2)}$. The latter are said to be commensurate if

$$
T_{\mathrm{cl}}^{(1)}=\frac{a}{b} T_{\mathrm{cl}}^{(2)}
$$


where $a$ and $b$ are relatively prime integers. When the classical periods are commensurate, the wave-packet displays a period on short time scales given by

$$
T_{\mathrm{cl}}=b T_{\mathrm{cl}}^{(1)}=a T_{\mathrm{cl}}^{(2)}
$$

If two classical periods are incommensurate, the initital motion of $\Psi(t)$ is not exactly periodic.

On larger time scales the three quantities $t_{\mathrm{rev}}^{(1)}, t_{\mathrm{rev}}^{(2)}$, and $t_{\mathrm{rev}}^{(12)}$ play a role in controlling the behavior, eventually inducing the wave-packet collapse. It follows from the form of the three second-order terms in the time-dependent phase that full revivals appear if the three revival times $t_{\mathrm{rev}}^{(1)}, t_{\mathrm{rev}}^{(2)}$, and $t_{\mathrm{rev}}^{(12)}$ are commensurate and satisfy

$$
t_{\mathrm{rev}}^{(1)}=\frac{c}{d} t_{\mathrm{rev}}^{(2)}=\frac{e}{f} t_{\mathrm{rev}}^{(12)}
$$

where $c, d$ and $e, f$ are pairs of relatively prime integers.

When Eq. (9) is obeyed, there is a time $t=t_{\text {rev }}$ at which all three second-order terms in the phase become integer multiples of $2 \pi$. Near this time, a full revival occurs: the phase is governed once more by the first-order terms, so the wave-packet motion and shape resemble those of the initial wave packet. Typically, the full revival time $t_{\mathrm{rev}}$ is a multiple of $t_{\mathrm{rev}}^{(1)}, t_{\mathrm{rev}}^{(2)}$, and $t_{\mathrm{rev}}^{(12)}$. However, it may equal one or more of the three component revival times for particular values of the integers in (9).

The appearance of fractional revivals requires that the wave function $\Psi(t)$ in Eq. (6) be expressible as a sum of subsidiary wave functions. This is possible only at times $t=t_{\text {frac }}$ that are simultaneously irreducible rational fractions of all three revival time scales. We therefore write the fractional-revival time scale as

$$
t_{\mathrm{frac}}=\frac{p_{1}}{q_{1}} t_{\mathrm{rev}}^{(1)}=\frac{p_{2}}{q_{2}} t_{\mathrm{rev}}^{(2)}=\frac{p_{12}}{q_{12}} t_{\mathrm{rev}}^{(12)}
$$

Here, the pairs of integers $\left(p_{1}, q_{1}\right),\left(p_{2}, q_{2}\right)$, and $\left(p_{12}, q_{12}\right)$ are relatively prime. Note that the condition (9) for the appearance of a full revival is automatically fulfilled if times $t_{\text {frac }}$ can be found that satisfy Eq. (10). The formation of full revivals is therefore a special case in the analysis below.

If a set of integers obeying (10) exists, then the issue of the appearance of fractional revivals at $t=t_{\text {frac }}$ becomes pertinent. To make further progress, we introduce a 
doubly-periodic function depending only on the classical time scales:

$$
\psi_{\mathrm{cl}}\left(t_{1}, t_{2}\right)=\sum_{k_{1}, k_{2}} c_{k_{1} k_{2}} \phi_{k_{1} k_{2}} \exp \left[-2 \pi i\left(\frac{k_{1} t_{1}}{T_{\mathrm{cl}}^{(1)}}+\frac{k_{2} t_{2}}{T_{\mathrm{cl}}^{(2)}}\right)\right] .
$$

Here, $t_{1}$ and $t_{2}$ are dummy variables. The function $\psi_{\mathrm{cl}}\left(t_{1}, t_{2}\right)$ is periodic in $t_{1}$ and $t_{2}$ with periods $T_{\mathrm{cl}}^{(1)}$ and $T_{\mathrm{cl}}^{(2)}$, respectively:

$$
\begin{aligned}
& \psi_{\mathrm{cl}}\left(t_{1}+T_{\mathrm{cl}}^{(1)}, t_{2}\right)=\psi_{\mathrm{cl}}\left(t_{1}, t_{2}\right) \\
& \psi_{\mathrm{cl}}\left(t_{1}, t_{2}+T_{\mathrm{cl}}^{(2)}\right)=\psi_{\mathrm{cl}}\left(t_{1}, t_{2}\right) .
\end{aligned}
$$

The functions $\psi_{\mathrm{cl}}\left(t_{1}, t_{2}\right)$ have no immediate physical significance. However, at the time $t=t_{1}=t_{2}$ the function $\psi_{\mathrm{cl}}(t, t)$ is equal to the wave function $\Psi(t)$ of Eq. (畐) when the second-order terms in the phase are omitted. Thus, the functions $\psi_{\mathrm{cl}}(t, t)$ exhibit the beating of $T_{\mathrm{cl}}^{(1)}$ and $T_{\mathrm{cl}}^{(2)}$. Note that $\Psi(0)=\psi_{\mathrm{cl}}(0,0)$, so for small times the behavior of $\Psi(t)$ is approximately matched by that of $\psi_{\mathrm{cl}}(t, t)$.

The functions $\psi_{\mathrm{cl}}\left(t_{1}, t_{2}\right)$ are useful because we can prove that, near the times $t_{\text {frac }}$ given in Eq. (10), the wave packet $\Psi(t)$ can be written as a sum of subsidiary waves $\psi_{\mathrm{cl}}$ with arguments shifted relative to $t$ by certain fractions of the corresponding periods. The proof involves examining the cyclic properties in $k_{1}$ and $k_{2}$ of the second-order terms in the time-dependent phase of $\Psi(t)$ and demonstrating that the functions $\psi_{\mathrm{cl}}$ with shifted arguments have identical cyclic properties. The result means that the $\psi_{\mathrm{cl}}$ with shifted arguments form an acceptable basis for the expansion of $\Psi(t)$ at the times $t_{\text {frac }}$.

To examine the cyclic properties in $k_{1}$ and $k_{2}$ of the second-order contributions to the time-dependent phase in $\Psi(t)$ at $t=t_{\text {frac }}$, we write these contributions as $\exp \left(-2 \pi i \theta_{k_{1} k_{2}}\right)$, where

$$
\theta_{k_{1} k_{2}}=\frac{p_{1}}{q_{1}} k_{1}^{2}+\frac{p_{2}}{q_{2}} k_{2}^{2}+\frac{p_{12}}{q_{12}} k_{1} k_{2}
$$

We seek the minimum periods $l_{1}$ and $l_{2}$ such that

$$
\theta_{k_{1}+l_{1}, k_{2}}=\theta_{k_{1} k_{2}} \quad, \quad \theta_{k_{1}, k_{2}+l_{2}}=\theta_{k_{1} k_{2}}
$$

Note that the relations

$$
t_{\mathrm{rev}}^{(1)}=\frac{r_{1}}{s_{1}} t_{\mathrm{rev}}^{(12)} \quad, \quad t_{\mathrm{rev}}^{(2)}=\frac{r_{2}}{s_{2}} t_{\mathrm{rev}}^{(12)}
$$


follow from (10) with

$$
\frac{r_{1}}{s_{1}}=\frac{q_{1} p_{12}}{p_{1} q_{12}} \quad, \quad \frac{r_{2}}{s_{2}}=\frac{q_{2} p_{12}}{p_{2} q_{12}} .
$$

The periods $l_{1}$ and $l_{2}$ must then satisfy

$$
\begin{aligned}
& \frac{p_{1}}{q_{1}} l_{1}^{2}+\frac{2 p_{1}}{q_{1}} l_{1} k_{1}+\frac{p_{1} r_{1}}{q_{1} s_{1}} l_{1} k_{2}=0 \quad(\bmod 1) \\
& \frac{p_{2}}{q_{2}} l_{2}^{2}+\frac{2 p_{2}}{q_{2}} l_{2} k_{2}+\frac{p_{2} r_{2}}{q_{2} s_{2}} l_{2} k_{1}=0 \quad(\bmod 1) .
\end{aligned}
$$

These relations can be satisfied by choosing $l_{1}=q_{1} s_{1}$ and $l_{2}=q_{2} s_{2}$. However, smaller values might exist if cancellations occur in some ratios.

The second-order contributions $\theta_{k_{1} k_{2}}$ are cyclic in $k_{1}$ and $k_{2}$ with periods $l_{1}$ and $l_{2}$, respectively. The functions $\psi_{\mathrm{cl}}\left(t+s_{1} T_{\mathrm{cl}}^{(1)} / l_{1}, t+s_{2} T_{\mathrm{cl}}^{(2)} / l_{2}\right)$ with shifted arguments have the same periodicities in $k_{1}$ and $k_{2}$. Here, $\psi_{\mathrm{cl}}$ is defined in Eq. (11) and $s_{1}=$ $0,1, \ldots, l_{1}-1$ and $s_{2}=0,1, \ldots, l_{2}-1$. This suggests the set of functions $\psi_{\mathrm{cl}}$ with shifted arguments can be used as a basis for an expansion of $\Psi(t)$ near the time $t_{\text {frac }}$.

Explicitly, the wave packet at the times $t \approx t_{\text {frac }}$ can be written as

$$
\Psi(t)=\sum_{s_{1}=0}^{l_{1}-1} \sum_{s_{2}=0}^{l_{2}-1} a_{s_{1} s_{2}} \psi_{\mathrm{cl}}\left(t+\frac{s_{1}}{l_{1}} T_{\mathrm{cl}}^{(1)}, t+\frac{s_{2}}{l_{2}} T_{\mathrm{cl}}^{(2)}\right) .
$$

The integers $l_{1}$ and $l_{2}$ depend on the values of $t_{\text {frac }}$. They are obtained by solving the conditions (18) and (19). The coefficients $a_{s_{1} s_{2}}$ are given by

$$
a_{s_{1} s_{2}}=\frac{1}{l_{1} l_{2}} \sum_{\kappa_{1}=0}^{l_{1}-1} \sum_{\kappa_{2}=0}^{l_{2}-1} \exp \left(-2 \pi i \theta_{\kappa_{1} \kappa_{2}}\right) \exp \left(2 \pi i \frac{s_{1}}{l_{1}} \kappa_{1}\right) \exp \left(2 \pi i \frac{s_{2}}{l_{2}} \kappa_{2}\right) \text {, }
$$

where $\theta_{\kappa_{1} \kappa_{2}}$ is given by Eq. (14). If the expressions for $a_{s_{1} s_{2}}$ and the $\psi_{\mathrm{cl}}$ are substituted into Eq. (20), the expansion reduces to Eq. (6) evaluated at the time $t=t_{\text {frac }}$, which completes the proof.

The expansion in (20) shows that the wave packet can be written as a sum of subsidiary wave functions at $t \approx t_{\text {frac }}$. However, it can be seen from Eq. (20) that the subsidiary wave functions typically have distinct shifts in the two arguments and hence do not follow the initial evolution of $\Psi(t)$, which is given by $\psi_{\mathrm{cl}}(t, t)$. Nonetheless, if near these times the shifted subsidiary wave functions are macroscopically distinct 
and localized in space, then $\Psi(t)$ has a periodicity that is a fraction of the classical time periods.

The detailed behavior of the fractional revivals is governed by the form of the expansion coefficients $a_{s_{1} s_{2}}$ given in Eq. (21). These coefficients depend on the function $\theta_{k_{1} k_{2}}$ defined in Eq. (14), which in turn depends on the commensurability of the three component revival times. An interesting special case occurs if the crossderivative term in the energy expansion vanishes, $\partial^{2} E / \partial n_{1} \partial n_{2}=0$, whereupon there is no cross-revival time $t_{\mathrm{rev}}^{(12)}$. The coefficients $a_{s_{1} s_{2}}$ can then be written as a product $a_{s_{1}}^{(1)} a_{s_{2}}^{(2)}$, where $a_{s_{1}}^{(1)}$ and $a_{s_{2}}^{(2)}$ each separately have the form of expansion coefficients for the conventional fractional revivals [6]. Nonvanishing values of $a_{s_{1}}^{(1)}$ acquire the same norm for all $s_{1}$. The same holds for $a_{s_{2}}^{(2)}$. This means that for zero cross-revival time the coefficients $a_{s_{1} s_{2}}$ are equal to a product of the corresponding coefficients for systems with conventional fractional revivals. Nonetheless, the function $\psi_{\mathrm{cl}}$ in Eq. (11) cannot be separated into a product of one-dimensional functions unless there is no entanglement of the states in the superposition. Only in this special case can the fractional revivals for systems with energies that depend on two quantum numbers be written as a product of two conventional fractional revivals. We refer to superpositions having these special properties as totally separable wave packets. For such packets, it follows that if $N_{1}$ subsidiary wave packets appear for one component while $N_{2}$ appear for the other, then the fractional revival for the full system consists of $N_{1} N_{2}$ subsidiary wave packets.

If the cross-revival time $t_{\mathrm{rev}}^{(12)}$ is nonzero, the expansion coefficients $a_{s_{1} s_{2}}$ cannot be written as a product $a_{s_{1}}^{(1)} a_{s_{2}}^{(2)}$. Their form is more complicated, and typically they have different norms for distinct $s_{1}$ and $s_{2}$. The fractional revivals are therefore less likely to cause distinctive periodicities in the autocorrelation function. However, for certain circumstances only a small number of subsidiary wave functions enter the expansion. This can happen, for example, if the values of $l_{1}$ and $l_{2}$ are small or if some of the $a_{s_{1} s_{2}}$ coefficients vanish. In this case, fractional periodicities should appear in the autocorrelation function.

Typically, the conditions (10) for the existence of a time $t_{\text {frac }}$ at which fractional revivals can occur are quite restrictive. For most values of $t_{\mathrm{rev}}^{(1)}, t_{\mathrm{rev}}^{(2)}$, and $t_{\mathrm{rev}}^{(12)}$, no 
solution exists. However, for many systems with energies that depend on two quantum numbers and that are of experimental interest, there is at least one tunable parameter such as a field strength or a length scale. If the energy depends on the tunable parameter, then so in general do the component revival times. It may therefore be feasible to tune the system so that a particular fractional revival exists. Since only certain commensurabilities are likely to hold, a partial spectrum of fractional revivals should be seen. For different values of the tunable parameter, we expect different partial spectra of fractional revivals.

As an example of a totally separable wave packet, consider a superposition of energy states for a particle in a two-dimensional box with periodic boundary conditions. The box is taken to have lengths $L_{1}$ and $L_{2}$ in the $x$ and $y$ directions, respectively, and the mass of the particle is chosen to be that of the electron, i.e., one in atomic units. The eigenfunctions are separable in $x$ and $y$, with eigenenergies $E_{n_{1} n_{2}}=2 \pi^{2}\left(n_{1}^{2} L_{1}^{-2}+n_{2}^{2} L_{2}^{-2}\right)$. The wave packet is taken as a superposition of states centered on $\bar{n}_{1}$ and $\bar{n}_{2}$ with weighting coefficients $c_{n_{1} n_{2}}=c_{n_{1}}^{(1)} c_{n_{2}}^{(2)}$, where $\left|c_{n_{1}}^{(1)}\right|^{2}$ and $\left|c_{n_{2}}^{(2)}\right|^{2}$ are both gaussian functions with widths $\sigma_{1}$ and $\sigma_{2}$, respectively.

Since the energy separates, there is no cross-revival time. Furthermore, since more than two derivatives of $E_{n_{1} n_{2}}$ vanish identically, there are no superrevivals for this system. This means the full revivals are perfect revivals for which the absolute square of the autocorrelation function is one [7]. The only nonzero revival times are $T_{\mathrm{cl}}^{(1)}=$ $L_{1}^{2} / 2 \pi \bar{n}_{1}, T_{\mathrm{cl}}^{(2)}=L_{2}^{2} / 2 \pi \bar{n}_{2}, t_{\mathrm{rev}}^{(1)}=L_{1}^{2} / \pi$, and $t_{\mathrm{rev}}^{(2)}=L_{2}^{2} / \pi$. The commensurability of the revival times may be tuned by adjusting the ratio $L_{1}^{2} / L_{2}^{2}$.

For definiteness, consider a superposition with $\bar{n}_{1}=\bar{n}_{2}=18$ in a system with $L_{1}=\sqrt{3} / 2$ and $L_{2}=1$. The ratio of the revival times is then $t_{\mathrm{rev}}^{(1)} / t_{\mathrm{rev}}^{(2)}=3 / 4$. We expect a full revival at $t=t_{\mathrm{rev}}=4 t_{\mathrm{rev}}^{(1)}=3 t_{\mathrm{rev}}^{(2)}=3 / \pi \simeq 0.95$ in atomic units. Figure 1 shows the absolute square of the autocorrelation function $|A(t)|^{2}=|\langle\Psi(0) \mid \Psi(t)\rangle|^{2}$ as a function of time. A perfect full revival is evident at $t=t_{\mathrm{rev}}$. At $t=t_{\mathrm{rev}}^{(1)} \simeq 0.24$ a.u. and $t=3 t_{\mathrm{rev}}^{(1)} \simeq 0.72$ a.u., corresponding to the choices $p_{1} / q_{1}=1, p_{2} / q_{2}=3 / 4$, and $p_{1} / q_{1}=3, p_{2} / q_{2}=9 / 4$, respectively, two subsidiary wave functions form. At these times $|A(t)|^{2}=1 / 2$, as can be seen in Fig. 1. At $t=2 t_{\mathrm{rev}}^{(1)} \simeq 0.48$ a.u., corresponding to $p_{1} / q_{1}=2$ and $p_{2} / q_{2}=3 / 2$, one wave function forms in each component. However, 
this time $t=t_{\mathrm{rev}}^{(2)} / 2$, which means the second-component subsidiary wave is a half cycle out of phase. Thus, as seen in Fig. 1, the autocorrelation $A(t)$ is approximately zero at this time.

As a second explicit example, set $L_{1}=1$ and $L_{2}=\sqrt{3}$, keeping $\bar{n}_{1}=\bar{n}_{2}=18$. These dimensions produce the ratio $t_{\mathrm{rev}}^{(1)} / t_{\mathrm{rev}}^{(2)}=1 / 3$, and as a result different types of fractional revivals will occur. The autocorrelation function for this case is shown in Fig. 2. A perfect full revival occurs at $t=t_{\mathrm{rev}}=3 t_{\mathrm{rev}}^{(1)}=4 t_{\mathrm{rev}}^{(2)}=3 / \pi \simeq 0.95$ in atomic units. Fractional revivals consisting of three subsidiary wave functions form at multiples of $t_{\text {rev }}^{(1)} / 2 \simeq 0.16$ a.u., with the exception of the time $3 t_{\text {rev }}^{(1)} / 2$. At this time, $p_{1} / q_{1}=3 / 2$ and $p_{2} / q_{2}=1 / 2$, and each component consists of a single wave packet forming one-half cycle out of phase with the classical motion. This does not result in a perfect revival because the cycles are not equal, $T_{\mathrm{cl}}^{(1)} / 2 \neq T_{\mathrm{cl}}^{(2)} / 2$, so the two components are out of phase. Additional fractional revivals consisting of four subsidiary wave functions are visible at times $t=3 t_{\text {rev }}^{(1)} / 4 \simeq 0.24$ a.u. and $t=9 t_{\mathrm{rev}}^{(1)} / 4 \simeq 0.72$ a.u.

The analysis in this paper is experimentally testable. As an explicit example of a physical system for which the energy depends on two quantum numbers and a crossrevival time exists, we can consider a Stark wave packet. This type of packet can be produced using a static electric field applied to an atom, which shifts and splits the energy levels. Applying a short laser pulse then creates a coherent superposition of Stark levels. The energies in atomic units for hydrogen and a weak electric field are $E_{n k}=-1 / 2 n^{2}+3 n k F / 2$, where $n$ is the principal quantum number, $k$ is the difference $n_{1}-n_{2}$ between the parabolic quantum numbers, and $F$ is the magnitude of the electric-field strength.

Stark wave packets have unique features because the quantum number $k$ is even or odd according to whether $n$ is odd or even and because for fixed $n$ the adjacent $k$ values differ by two units. These properties introduce some additional complications in the analysis of fractional revivals and produce novel interference patterns in the interferograms of Stark wave packets [18]. A second-order Taylor expansion of the energy $E_{n k}$ with respect to $n$ and $k$ can still be performed, but some changes occur in the definitions of the controlling time scales. Since the quantum number $k$ is either 
even or odd, a Stark wave packet effectively evolves as two separate wave packets. At the fractional revival times, the packet separates into sums over sets of subsidiary wave functions consisting of superpositions of even- $n$ and odd- $n$ states. These wave functions evolve with distinct relative phases and obey distinct periodicity relations. This results in additional interference patterns in the autocorrelation function, which can be tested experimentally.

One of us (R.B.) would like to thank Colby College for a Science Division grant. This work is supported in part by the National Science Foundation under grant number PHY-9503756.

\section{REFERENCES}

1. J.A. Yeazell and C.R. Stroud, Phys. Rev. A 43, 5153 (1991).

2. D.R. Meacher, P.E. Meyler, I.G. Hughes, and P. Ewart, J. Phys. B 24, L63 (1991).

3. J. Wals, H.H. Fielding, J.F. Christian, L.C. Snoek, W.J. van der Zande, and H.B. van Linden van den Heuvell, Phys. Rev. Lett. 72, 3783 (1994).

4. M.J.J. Vrakking, D.M. Villeneuve, and A. Stolow, Phys. Rev. A 54, R37 (1996).

5. G. Alber, H. Ritsch, and P. Zoller, Phys. Rev. A 34, 1058 (1986); G. Alber and P. Zoller, Phys. Rep. 199, 231 (1991).

6. I.Sh. Averbukh and N.F. Perelman, Phys. Lett. 139A, 449 (1989).

7. R. Bluhm, V.A. Kostelecký, and J. Porter, Am. J. Phys. 64, 944 (1996) (quantph/9510029).

8. R. Bluhm and V.A. Kostelecký, Phys. Rev. A 50, R4445 (1994) (hepph/9410325); Phys. Lett. A 200, 308 (1995) (quant-ph/9508024); Phys. Rev. A 51, 4767 (1995) quant-ph/9506009).

9. J. Parker and C.R. Stroud, Phys. Rev. Lett. 56, 716 (1986).

10. A. Peres, Phys. Rev. A 47, 5196 (1993). 
11. V.A. Kostelecký and M.M. Nieto, Phys. Rev. Lett. 53, 2285 (1984); Phys. Rev. A 32, 1293, 3243 (1985).

12. R. Bluhm and V.A. Kostelecký, Phys. Rev. A 48, R4047 (1993) (quantph/9508019); Phys. Rev. A 49, 4628 (1994) (quant-ph/9508020); R. Bluhm, V.A. Kostelecký, and B. Tudose, Phys. Rev. A 52, 2234 (1995) quantph/9509010); Phys. Rev. A 53, 937 (1996) (quant-ph/9510023).

13. A. ten Wolde, L.D. Noordam, A. Lagendijk, and H.B. van Linden van den Heuvell, Phys. Rev. A 40, 485 (1989); L.D. Noordam, A. ten Wolde, A. Lagendijk, and H.B. van Linden van den Heuvell, Phys. Rev. A 40, 6999 (1989).

14. J.A. Yeazell, G. Raithel, L. Marmet, H. Held, and H. Walther, Phys. Rev. Lett. 70, 2884 (1993).

15. B. Broers, J.F. Christian, J.H. Hoogenraad, W.J. van der Zande, H.B. van Linden van den Heuvell, and L.D. Noordam, Phys. Rev. Lett. 71, 344 (1993).

16. G.M. Lankhuijzen and L.D. Noordam, Phys. Rev. A 52, 2016 (1995).

17. M.L. Naudeau, C.I. Sukenik, and P.H. Bucksbaum, "Core Scattering of Stark Wavepackets," University of Michigan preprint.

18. R. Bluhm, V.A. Kostelecký, and B. Tudose, Phys. Rev. A, in press quantph/9608040). 


\section{FIGURE CAPTIONS}

Fig. 1: The absolute square of the autocorrelation function is plotted as a function of time in atomic units for a particle in a two-dimensional box with $\bar{n}_{1}=\bar{n}_{2}=18$, $L_{1}=\sqrt{3} / 2$, and $L_{2}=1$.

Fig. 2: The absolute square of the autocorrelation function is plotted as a function of time in atomic units for a particle in a two-dimensional box with $\bar{n}_{1}=\bar{n}_{2}=18$, $L_{1}=1$, and $L_{2}=\sqrt{3}$. 